\title{
Machine-learning semilocal density functional theory for many-body lattice models at zero and finite temperature
}

\author{
James Nelson $\odot,{ }^{*}$ Rajarshi Tiwari $\odot,{ }^{\dagger}$ and Stefano Sanvito $\odot^{\dagger}$ \\ School of Physics, AMBER and CRANN Institute, Trinity College, Dublin 2, Ireland
}

(Received 10 March 2021; accepted 18 May 2021; published 7 June 2021)

\begin{abstract}
We introduce a machine-learning density-functional-theory formalism for the spinless Hubbard model in one dimension at both zero and finite temperature. In the zero-temperature case this establishes a one-to-one relation between the site occupation and the total energy, which is then minimized at the ground-state occupation. In contrast, at finite temperature the same relation is defined between the Helmholtz free energy and the equilibrium site occupation. Most importantly, both functionals are semilocal, so that they are independent from the size of the system under investigation and can be constructed over exact data for small systems. These "exact" functionals are numerically defined by neural networks. We also define additional neural networks for finite-temperature thermodynamical quantities, such as the entropy and heat capacity. These can be either a functional of the ground-state site occupation or of the finite-temperature equilibrium site occupation. In the first case their equilibrium value does not correspond to an extremal point of the functional, while it does in the second case. Our work gives us access to finite-temperature properties of many-body systems in the thermodynamic limit.
\end{abstract}

DOI: 10.1103/PhysRevB.103.245111

\section{INTRODUCTION}

Machine learning (ML) is a wide field of computer science. It is constructed over a collection of algorithms and numerical techniques and aims at recognizing and manipulating the patterns hidden in large volumes of data [1]. Although the most impressive progress in the field is related to signal processing (images, videos, etc.), ML is now becoming a powerful tool in both experimental and computational materials science and in engineering [2,3]. Examples of applications include the design of new molecules, [4] the running of self-driving labs $[5,6]$, the construction of ultra-accurate force fields $[7,8]$, the prediction of physical properties based on structure-toproperty [9] or chemistry-to-property [10] relations, and the analysis of data from electron microscopy [11], just to name a few.

In addition to materials science and engineering the use of ML is now widely spread over an impressive range of problems in Physics [12]. In particular, it has been utilized to address the complexity of a number of many-body models. For these an exact solution typically does not exist, except for a few limiting cases, while exact diagonalization is bound by the severe scaling of the Hilbert space with the system size, and hence by the computational costs. For instance, ML has been used to find ground-state properties and observables of fermion-boson coupled Hamiltonians [13], disordered Hubbard-Anderson models [14] and a variety of spin models [15-17]. At the same time some progress has been

\footnotetext{
*janelson@tcd.ie

†tiwarir@tcd.ie

‡stefano.sanvito@tcd.ie
}

made in classifying quantum states at finite temperature and thus identifying phase transitions $[18,19]$. Finally, restricted Boltzmann machines have been constructed to represent and propagate in time wavefunctions [20], a work that has stimulated the introduction of open-source software for the study of many-body models [21].

Several numerical methods have been designed to solve many-body problems for systems, whose size makes them not accessible by exact diagonalization. If the interest lies in ground-state properties, density matrix renormalization group [22] and variational Monte Carlo [23] methods are valuable options, while at finite temperature continuous-time Monte Carlo [24] and dynamic mean field theory [25] can be considered. These methods, depending on the nature of their implementation/design, usually suffer from (i) restrictions to low or high dimensions, (ii) the fermionic sign problem, (iii) poor reliability at either low or high temperatures. In any case, although some schemes may allow one to investigate relatively large systems, their numerical overheads are still significant. This means that information concerning the thermodynamic limit of the various models and about the interplay between interaction and disorder remain difficult to access.

In this context functional-based methods, such as density functional theory (DFT), deserve a class on their own. These are based on the two Hohenberg-Kohn theorems [26], which have also been extended to lattice models [27-29]. The core idea is that the total energy can be expressed as a universal functional of the single-particle electron density, or its lattice equivalent, and that such functional has a minimum at the ground-state density, where it gives the ground-state energy. For interacting theories the universal functional is not known, although excellent approximations exist and a number of exact 
constraints have been rigorously proved. Most importantly for the discussion here, the functional formulation of the manybody problem appears ideal for a ML approach. In fact, one can construct a dataset of either exact or approximated results and try to learn the one-to-one relation between the electron density and the energy. Several examples of this approach exist for the actual Coulomb-interaction DFT [30] for both the noninteracting $[31,32]$ and interacting $[33,34]$ case in one dimension, and in two dimensions for selected external potentials $[35,36]$. There are also attempts in three dimensions, in particular, at constructing maps between the potential and the electron density [37] and between the electron density and the total energy [38,39], and in learning the self-consistent electron density in Kohn-Sham DFT [40].

In our previous work we have constructed an exact machine-learning DFT functional for the one-dimension Hubbard model using neural networks [41], a strategy also used by Moreno et al. to reconstruct the ground-state wavefunction [42]. We have then demonstrated that such functional satisfies both the Hohenberg-Kohn theorems [26]. The network, trained over exact-diagonalization results for systems defined by a random single-particle potential, establishes a one-to-one relation between the sites occupation and the ground-state total energy. Since the site occupation of every site (the total charge density) is used to define the functional, one has to construct a new network, namely, a new functional, for every different system size and every different filling factor. This means that, although the functional is exact to numerical precision it is of little practical use.

Here we circumvent the problem by constructing a semilocal functional, which requires only local knowledge of the site occupation. This can now be trained over small systems and be used to predict the ground-state properties of systems of any size. The same formalism is then extended to finite-temperature, a task that is approached in two different ways. First, by using one of the results of the HohenbergKohn theorems, we construct neural networks that relate the ground-state density to the thermal average of a number of operators. These define the finite-temperature equilibrium site occupation, energy, entropy and specific heat. In this case, although such quantities are functional of the ground-state site occupation, they are not found at the functional minimum, namely, they cannot be determined by variational principle. In contrast, the second approach consists in extending DFT to the canonical ensemble as proposed a long time ago by Mermin [43]. The one-to-one correspondence is now between the finite-temperature equilibrium site occupation and the Helmholtz free energy. Also in this case we construct a universal semilocal functional, which is minimized at the equilibrium site occupation.

The paper is organized as follows. In the next section we introduce our ML models, by presenting the semilocal functional and the representation used for the site occupation, by introducing functionals for finite-temperature thermodynamic properties and by formulating the extension of DFT to the canonical ensemble. In this section we will also discuss the details of the neural networks constructed. Then we present our results focusing first on the zero-temperature limit and then on its finite-temperature extension. In particular, we discuss the actual level of nonlocality required by the functionals and analyze the homogeneous limit. Finally, we show how the functional can be minimized to find the ground-state density (or the finite temperature equilibrium density) and how this procedure allows us to extract finite-temperature properties of the model for systems of any size. Finally, we conclude.

\section{CONSTRUCTION OF THE MODELS}

\section{A. Zero-temperature lattice DFT: The semilocal density approximation}

Our analysis applies to any lattice model with Hamiltonian of the form

$$
\hat{H}=\hat{H}_{0}+\hat{H}_{\text {kin }}+\hat{H}_{\text {int }},
$$

where $\hat{H}_{0}$ is a single-particle potential, $\hat{H}_{\text {kin }}$ is the kinetic energy and $\hat{H}_{\text {int }}$ is some form of many-body interaction. In particular, here we focus our attention on the spinless nonlocal Hubbard model in one dimension. This is implemented with periodic boundary conditions, namely, for finite rings comprising $L$ sites. The corresponding Hamiltonian, $\hat{H}_{V}$, thus reads

$$
\hat{H}_{V}=\sum_{i=1}^{L} \epsilon_{i} \hat{n}_{i}-t \sum_{i=1}^{L}\left(\hat{c}_{i}^{\dagger} \hat{c}_{i+1}+\hat{c}_{i+1}^{\dagger} \hat{c}_{i}\right)+V \sum_{i=1}^{L} \hat{n}_{i+1} \hat{n}_{i},
$$

where $\hat{c}_{i}^{\dagger}\left(\hat{c}_{i}\right)$ is the creation (annihilation) operator for a spinless electron at site $i, \hat{n}_{i}=\hat{c}_{i}^{\dagger} \hat{c}_{i}$ is the number operator, $\epsilon_{i}$ are the onsite energies, $V$ is the nonlocal Hubbard parameter and $t=1$ is the hopping integral that sets the energy scale of the problem. The results presented here are for the half-filling case, namely, the total number of electrons is $N_{e}=L / 2$.

Since we are going to compare quantities for lattices of different size, it is convenient to define any observable in terms of its density, namely, by dividing it by the number of lattice sites. For instance, the energy density is simply, $e \equiv E / L$, where $E$ is the total energy. It was demonstrated some time ago that the Hohenberg-Kohn theorem [26] can be extended to lattice models [27-29], once the appropriate single-particle density is defined. In the case of the spinless Hubbard model the fundamental quantity is the sites occupation, $\left\{n_{i}\right\}$, defined as the expectation value of the number operator, $n_{i}=\left\langle\hat{n}_{i}\right\rangle$, over all the sites. As a matter of notation here we represent the ground-state expectation value of the generic operator $\hat{O}$ as $\left\langle\Psi_{0}|\hat{O}| \Psi_{0}\right\rangle=\langle\hat{O}\rangle_{0}$, where $\Psi_{0}$ is the many-body ground-state wave-function. The energy density functional (in this case an actual function) can then be written as

$$
e\left[\left\{n_{i}\right\}\right]=f_{V}\left[\left\{n_{i}\right\}\right]+\frac{1}{L} \sum_{i=1}^{L} \epsilon_{i} n_{i},
$$

where $f_{V}\left[\left\{n_{i}\right\}\right]$ is a universal functional of $\left\{n_{i}\right\}$ and it is defined for every value of $V$ (there is a functional for every value of $V)$. In our previous work we have shown that a numerically exact functional could be learnt by using ML [41]. This satisfies both the two Hohenberg-Kohn theorems, namely, for a given ground-state density it yields the external potential, and it is variational in the site occupation. The second statement implies that the minimum of the functional is found at the ground-state site occupation, $n_{i}^{0}=\left\langle\hat{n}_{i}\right\rangle_{0}$, where it returns the ground-state energy density, $e^{0}=e\left(\left\{n_{i}^{0}\right\}\right)$. Such ML 


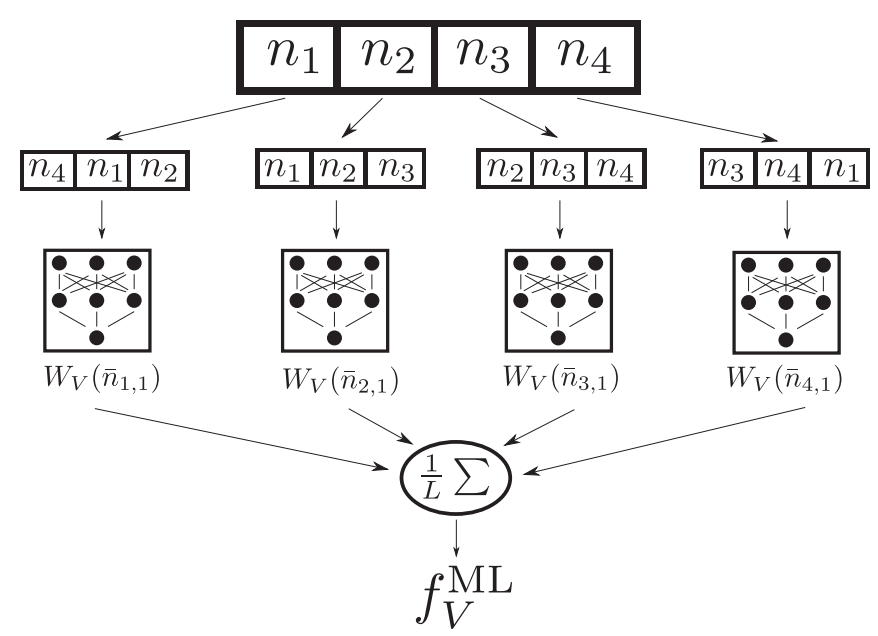

FIG. 1. An illustration of how the semilocal functional, $f_{V}^{\mathrm{ML}}\left[\left\{n_{i}\right\}\right]$, is constructed for a four-site ring with $a=1$. In this case four occupations, $\left\{n_{1}, n_{2}, n_{3}, n_{4}\right\}$, define uniquely the total energy. This is written as the site average average of four $W_{V}\left(\bar{n}_{i, a}\right)$ contributions, constructed via a ML neural network, each one of them depending only on three site occupations.

functional depends on the sites occupation at every site; therefore, it is strictly defined for a particular system size and electron filling factor. Thus, the functional is of little practical use, since it needs to be constructed for any specific system under investigation, an operation that is limited by the computational ability of solving exactly the full many-body problem.

Here we overcome such drawback by introducing a new semilocal functional that is, by construction, lattice-size independent. This is defined as

$$
f_{V}^{\mathrm{ML}}\left[\left\{n_{i}\right\}\right]=\frac{1}{L} \sum_{i=1}^{L} W_{V}\left(\bar{n}_{i, a}\right),
$$

where $W_{V}\left(\bar{n}_{i, a}\right)$ is the energy associated to the $i$-th site. In turn, $W_{V}\left(\bar{n}_{i, a}\right)$ depends on the "local" site occupation,

$$
\bar{n}_{i, a}=\left\{n_{i-a}, n_{i-a+1}, \ldots, n_{i}, \ldots, n_{i+a-1}, n_{i+a}\right\},
$$

meaning that the energy associated to site $i$ depends on the occupation at site $i$ and on that at the first $a$ sites around it (overall, it depends on the site occupation at $2 a+1$ sites). Thus $a$ defines the locality of the functional, $f_{V}^{\mathrm{ML}}\left[\left\{n_{i}\right\}\right]$, which is simply the average of $W_{V}\left(\bar{n}_{i, a}\right)$ over all the sites. Figure 1 illustrates an example of how to construct the functional for a ring of 4 sites and a local density of range $a=1$.

Recently, a conceptually similar way to construct ML models for extensive quantities has been brought forward by Mills and coworkers, [44] who represented the physical space of the model (e.g., the atomic sites) across a set of nonoverlapping regions. A common neural network defined for such region is then averaged over the entire space to yield the desired extensive quantity. Our approach is similar in spirit, with the main difference being the use of a site-centered representation of the local quantity of interest (the site occupation). As well as being lattice-size independent this approximation has site symmetry automatically built into it, namely, it will predict, by construction, the same energy for densities formed by translating the onsite potential, $\left\{\epsilon_{i}\right\} \rightarrow\left\{\epsilon_{i+1}\right\}$.

\section{B. Zero-temperature lattice DFT: Thermodynamic quantities}

In lattice DFT (Coulomb DFT) the Hohenberg-Kohn theorem establishes a one-to-one correspondence between the ground-state site occupation (the single-particle electron density) and the onsite energies (the external potential). It follows that $\left\{n_{i}^{0}\right\}$ completely determines the Hamiltonian of the system, hence its solution. Thus, one can conclude that the entire many-body excitation spectrum is a functional (unknown) of the ground-state site occupation, namely,

$$
\left|\Psi_{m}\right\rangle=\left|\Psi_{m}\left[\left\{n_{i}^{0}\right\}\right]\right\rangle, \quad E_{m}=E_{m}\left[\left\{n_{i}^{0}\right\}\right], \quad \text { for all } m,
$$

where $E_{m}$ is the energy of the $m$ th many-body state and $\left|\Psi_{m}\right\rangle$ the associated wave-function.

Now take a generic operator, $\hat{O}$, and calculate its thermal average, $\langle\hat{O}\rangle_{T}$, in the canonical ensemble. This reads

$$
\langle\hat{O}\rangle_{T}=\sum_{n}\left\langle\Psi_{n}|\hat{O}| \Psi_{n}\right\rangle \frac{e^{-\beta E_{n}}}{Z},
$$

where $Z=\sum_{n} e^{-\beta E_{n}}$ is the partition function, and $\beta=1 / k_{\mathrm{B}} T$ with $k_{\mathrm{B}}$ being the Boltzmann constant and $T$ the temperature. It then follows, from Eq. (6), that also $\langle\hat{O}\rangle_{T}$ is a functional of the ground-state density, $\langle\hat{O}\rangle_{T}\left[\left\{n_{i}^{0}\right\}\right]$. Clearly in this case the variational principle is not established, meaning that $\langle\hat{O}\rangle_{T}$ does not necessarily have an extremal point at $\left\{n_{i}^{0}\right\}$. However, one can still define a map, $f$, between ground-state site occupation and temperature, and $\langle\hat{O}\rangle_{T}$,

$$
f:\left[\left\{n_{i}^{0}\right\}, T\right] \longrightarrow\langle\hat{O}\rangle_{T}
$$

We have thus constructed a number of neural networks to numerically estimate such mapping. These are also built within the semilocal approximation, namely, they have the form

$$
\langle\hat{o}\rangle_{T}^{\mathrm{ML}}=\frac{1}{L} \sum_{i=1}^{L} W_{V}^{O}\left(\bar{n}_{i, a}^{0}, T\right),
$$

where $\hat{o}=\hat{O} / L$ is the operator density and where now the neural network, $W_{V}^{O}\left(\bar{n}_{i, a}^{0}, T\right)$, depends also on the temperature. In particular, we have considered the entropy density, $\hat{s}$, the energy density, $\hat{e}$, and the heat capacity density, $\hat{c}$, which read, respectively,

$$
\begin{array}{r}
\langle\hat{s}\rangle_{T}=-\frac{1}{L} \sum_{n} p_{n} \ln p_{n}=\frac{1}{L} \frac{\partial}{\partial T}\left(k_{\mathrm{B}} T \ln Z\right), \\
\langle\hat{e}\rangle_{T}=\frac{1}{L} \sum_{n}\left\langle\Psi_{n}|\hat{H}| \Psi_{n}\right\rangle \frac{e^{-\beta E_{n}}}{Z}=-\frac{1}{L} \frac{\partial \ln Z}{\partial \beta}, \\
\langle\hat{c}\rangle_{T}=\frac{\partial\langle e\rangle_{T}}{\partial T}=\frac{1}{L} \frac{1}{k_{\mathrm{B}} T^{2}} \frac{\partial^{2} \ln Z}{\partial \beta^{2}},
\end{array}
$$

where $p_{n}=e^{-\beta E_{n}} / Z$.

Note that in general the ground-state site occupations determine the energy only up to a constant, $E_{\alpha}$. A constant shift in the many-body energy eigenvalues, $E_{n} \rightarrow E_{n}+E_{\alpha}$, transforms the partition function as $Z \rightarrow Z \exp \left(-\beta E_{\alpha}\right)$, but leaves invariant both $\langle\hat{s}\rangle_{T}$ and $\langle\hat{c}\rangle_{T}$. The same is not true, however, for $\langle e\rangle_{0}$ and $\langle e\rangle_{T}$, so that one has to constrain the 
onsite energies to a constant. In our previous work [41] such constraint was imposed by setting one of the onsite energies to zero. Here, to preserve the symmetry of the single particle potential, we impose that the onsite energies have always zero mean. We implement such condition by shifting the energy eigenstates by $E_{\alpha}=-\left(N_{e} / L\right) \sum_{j} \epsilon_{j}$, since this transforms the total energy as $E \rightarrow E+N_{e} E_{\alpha}$.

\section{Finite-temperature lattice DFT}

In conventional DFT the Hohenberg-Kohn theorems can be generalized to both the canonical and the grand canonical ensemble [43]. Their translation to lattice DFT for the specific case of the spinless Hubbard model and the canonical ensemble reads as follows. One can define a universal functional, $g_{V}\left[\left\{n_{i}\right\}\right]$, independent from the one-site energies, $\left\{\epsilon_{i}\right\}$, such that

$$
h\left[\left\{n_{i}\right\}\right]=g_{V}\left[\left\{n_{i}\right\}\right]+\frac{1}{L} \sum_{i=1}^{L} \epsilon_{i} n_{i}
$$

is minimum and equal to the Helmholtz free-energy density, $h$, associated to $\left\{\epsilon_{i}\right\}$, when $\left\{n_{i}\right\}$ is the equilibrium site occupation in the presence of $\left\{\epsilon_{i}\right\}$. This essentially means that at the minimum of the functional one has

$$
h\left[\left\{n_{i}^{T}\right\}\right]=\langle\hat{e}\rangle_{T}-T\langle\hat{s}\rangle_{T},
$$

with the equilibrium site occupation defined as [see Eq. (7)],

$$
n_{i}^{T}=\left\langle\hat{n}_{i}\right\rangle_{T}=\sum_{n}\left\langle\Psi_{n}\left|\hat{n}_{i}\right| \Psi_{n}\right\rangle \frac{e^{-\beta E_{n}}}{Z} .
$$

Also in this finite-temperature extension of DFT the universal functional can be constructed by using a neural network and a semilocal approximation, namely, we can define

$$
g_{V}^{\mathrm{ML}}\left[\left\{n_{i}\right\}\right]=\frac{1}{L} \sum_{i=1}^{L} X_{V}\left(\bar{n}_{i, a}\right),
$$

which again has a variational minimum at $\left\{n_{i}^{T}\right\}$.

Finally, note that the equilibrium site occupation at site $i$ can be simply viewed as the thermal average of the number operator, $\hat{n}_{i}$. Hence, we can construct a machine-learning model that, given $n_{i}^{0}$ and $T$, returns $n_{i}^{T}$ [see Eq. (9)], namely,

$$
n_{i}^{T}=\left\langle\hat{n}_{i}\right\rangle_{T}^{\mathrm{ML}}=W_{V}^{n_{i}}\left(\bar{n}_{i, a}^{0}, T\right) .
$$

Since all the functionals constructed here are semilocal, we have now a tool to approach the study of thermodynamic quantities for a disordered many-body system in the limit $L \rightarrow \infty$. In fact we have two options. On the one hand, one can consider finite-temperature lattice DFT and compute, by variational principle, both $\left\{n_{i}^{T}\right\}$ and $h\left[\left\{n_{i}^{T}\right\}\right]$. On the other hand, we can use zero-temperature DFT to determine $\left\{n_{i}^{0}\right\}$ and then, by employing the machine-learning maps of Eq. (8), all the thermal averaged observables. Indeed, one can also construct a "hybrid" approach where $\left\{n_{i}^{T}\right\}$ is derived from $\left\{n_{i}^{0}\right\}$ via Eq. (17) and then used to determine the Helmholtz free energy.
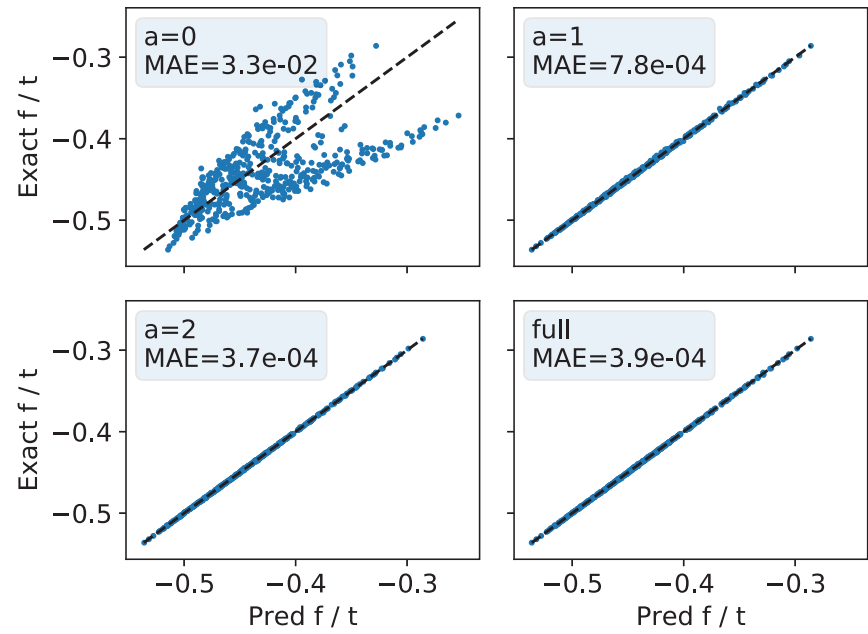

FIG. 2. Accuracy of the semilocal functional for ground-state DFT as a function of the locality parameter, $a$. Here we plot the value of the exact universal functional (computed from exact diagonalization) against the prediction obtained with our ML semilocal formulation. Results are for $L=6$ and $V=1$. The box inside each plot reports the $a$ value and the MAE, with "full" corresponding to a functional, where the entire site occupation vector is used (completely nonlocal case). Data are provided here for the test set and each graph contains 500 points.

\section{RESULTS}

To test the accuracy of our semilocal functionals we have generated datasets for $L=6,10,14$ and $V=1,2,4$, by exact diagonalization. The training and validation sets, used, respectively, to train the model and to enforce early stopping, contain data where the onsite energies are drawn from several uniform distributions in the interval, $[-W, W]$, with $W$ ranging from 2 to 8 . In total for any given $L$ and $V$ there are 24000 random onsite energy realizations in the training set and 8000 in the validation one. Finally, the test set contains 500 configurations generated for $W=4$.

\section{A. Construction of the semilocal functional at zero temperature}

To learn the zero-temperature universal functional, $f_{V}$, we have used a fully connected neural network, constructed by using PyTorch. [45] Weight sharing is implemented to ensure that the $L$ available local energies, $W_{V}\left\{\bar{n}_{(i, a)}\right\}$, are the same, and the "Adam" optimizer is chosen to update the models. The first validation of our strategy consists in investigating the accuracy of a functional constructed for a system of $L$ sites against new onsite energy configurations for systems with the same number of sites. Namely, we first check how the ML functional trained for $L$ performs against unknown $L$-site systems. This must be done as a function of the locality parameter, $a$, so that the efficacy of the semilocal approximation is established. Our results for $V=1$ and $L=6$ are shown in Fig. 2, where we plot the functional computed from exact diagonalization against our semilocal ML estimation for a collection of different systems. The figure of merit here is the mean absolute error (MAE) and similar trends have been observed for different values of $V$ and $L$. 

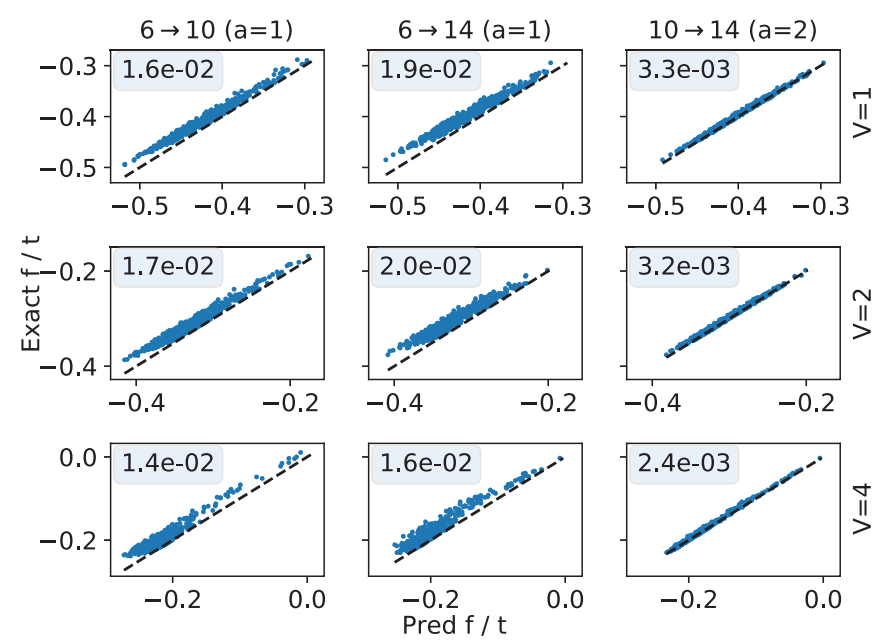

FIG. 3. Accuracy of the semilocal ground-state DFT functional trained for small systems and tested for larger ones as a function of the locality parameter, $a$. Here we plot the value of the exact universal functional (computed from exact diagonalization) against the prediction obtained with our ML semilocal formulation. In the first column the functionals have been trained on rings of $L=6$ sites and are used to predict $L=10$ data. The second column is for functionals trained on 6-site rings and predicting $L=14$ data. Finally, in the third column the functionals have been trained on 10 -site rings and used to predict $L=14$ data. The rows correspond to different values of $V$. The value of $a$ is the same for each column.

From the figure it is clear that, although a completely local approximation, $a=0$, is insufficient to approximate the functional, already a moderate degree on nonlocality, $a=1$, returns us a MAE just below $10^{-3}$, corresponding to an average error of the order of $0.2 \%$. Extending further the range of the functional improves the description, to a point that the $a=2$ case presents an error, $\sim 0.1 \%$, numerically indistinguishable from that of the fully nonlocal ML functional.

Since the local representation is lattice-size independent and the universal functional is semilocal, in principle one can use small, cheaply generated, systems as training set, and then predict quantities for larger systems. The results of this exercise are presented in Fig. 3, which compares the accuracy of ML functionals trained over rings of $L$ sites at predicting, $f_{V}$, for rings of $L^{\prime}$ sites (symbolically $L \rightarrow L^{\prime}$ ), with $L^{\prime}>L$ and for various values of $V$ and $a$. We can notice that when the ML training takes place over relatively small rings ( $L=6$ in this case) the ML estimate of $f_{V}$ appears to be larger in absolute value than the exact result, namely, all the points lie above the perfect-agreement line. This is, for instance, the case of training on a $L=6$ lattice and predicting for $L=10,14$. Such overestimation is systematic and independent from the interaction strength $V$. However, the error is immediately corrected when training the network on larger lattices and in fact the $10 \rightarrow 14$ case already offers an error below $1 \%$, with a nonlocality of $a=2$, regardless of $V$.

The inability to train the model over very small rings is rooted into our representation, which does not describe the homogeneous case. Consider, in fact, the situation where $\epsilon_{i}=$ $\epsilon_{0}$, namely, all the onsite energies are identical. Translational invariance then imposes that $n_{i}=N_{e} / L$ for any site $i$, so that $\bar{n}_{i, a}=\bar{n}_{a}=\left\{N_{e} / L, \ldots, N_{e} / L\right\}$. The ML functional then has the following simple form:

$$
f_{V}^{\mathrm{ML}}\left[\left\{n_{i}\right\}\right]=\frac{1}{L} \sum_{i=1}^{L} W_{V}\left(\bar{n}_{i, a}\right)=W_{V}\left(\bar{n}_{a}\right),
$$

namely, it returns the same energy density regardless of the system size, $e\left(\left\{n_{i}\right\}\right)=W_{V}\left(\bar{n}_{a}\right)+\epsilon_{0} N_{e} / L$. This is clearly incorrect, even for the noninteracting case $(V=0)$. Thus, our semilocal functional formulation is unable to describe the kinetic energy of rings of arbitrary size. Such error becomes progressively smaller as one trains the network on large rings, since the difference in kinetic energy density between a ring with $L$ sites and one with $L+n$ reduces with $L$. Eventually the error is completely eliminated in the thermodynamic limit, where the kinetic energy density becomes $4 t / \pi \sin \left(\frac{N_{e} \pi}{2 L}\right)$.

One can then design a number of alternative strategies to eliminate or mitigate the error made on the noninteracting kinetic energy density. For instance, we can explicitly subtract from the universal functional the noninteracting energy, a single-particle quantity easy to compute in a Kohn-Sham framework. This is effectively what usually done in conventional DFT, when defining the exchange and correlation functional. Here, we have decided not to include any correction, but simply train over larger rings (e.g., $L=10$ ), for which our numerical analysis demonstrates that the error is relatively small (see Fig. 3). Most importantly, it needs to be noted that the kinetic contribution to the total energy gets smaller as disorder is included (as we depart from the homogeneous limit) and the interaction gets larger.

The tests presented so far only concern the energy, namely, we have computed the value of $f_{V}$ at the exact charge density obtained with exact diagonalization. This simply demonstrates that our neural network is a good approximator for $f_{V}$, but does not mean that we have a variational theory. What we need to show instead is that a network trained on a small lattice can be minimized by the ground state charge density also for larger lattices, and that at the minimum the energy density returns its exact ground-state value. This essentially means that the functional is variational and it is transferrable from small to large rings (namely, the semilocal approximation works). Figure 4 provides such demonstration. By taking the $V=1$ case, we train a neural network on a $L=10$ ring. Then, by starting from the homogeneous site occupation distribution, $n_{i}=N_{e} / L$, we minimize the energy density for a ring of 14 sites. The minimization is performed by using gradient descent with momentum [1] (learning rate, 0.002, and momentum, 0.9), and then the converged site occupations are used to compute the energy density. The top panel of Fig. 4 compares the ML-computed energy density with the exact diagonalization result for 100 random rings. In the inset we show an histogram of the Euclidean distances between the initial and the converged site occupations, and the exact $\left\{n_{i}^{0}\right\}$. The agreement appears quite good, with a MAE of 0.0047 , and a generally accurate estimate for the ground-state occu- 

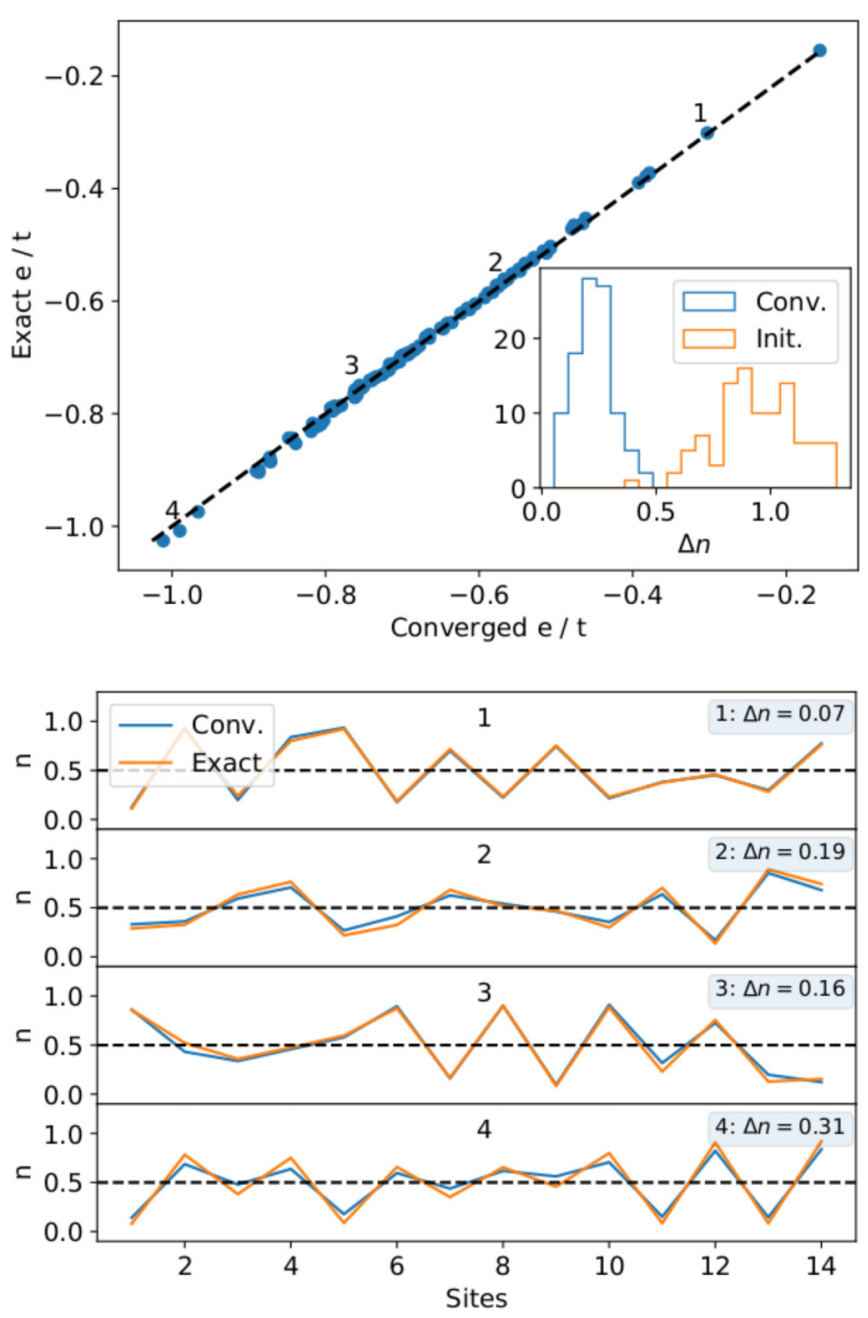

FIG. 4. Demonstration of the variational principle for the ML functional. In the top panel we show the energy density computed by minimization with respect to the site occupation. Our network has been trained for $L=10$ and used for a ring containing 14 sites $(V=1)$. The figure contains 100 different disorder realizations with $W=4$. The insert shows histograms of the Euclidean distances $\Delta n$, of the initial and converged site occupation from the exact one. The lower panel presents a selection of the converged occupations compared against the exact value. These are taken for different ground-state energy densities as indicated by the numbers in the upper panel. The overall MAE of the site occupation is 0.0047 .

pation. Some examples of converged site occupations are also included in the figure.

\section{B. Construction of the semilocal finite temperature maps}

In this section we establish one-to-one mappings between the ground-state occupation and a number of thermodynamic quantities. For this task we have used two fully connected neural networks; the first predicts the three scalar thermodynamic functions, $\langle\hat{s}\rangle_{T},\langle\hat{e}\rangle_{T}$, and $\langle\hat{c}\rangle_{T}$, and the second, the equilibrium site occupation $\left\{\left\langle n_{i}\right\rangle_{T}\right\}$ (a vector). For all of the thermodynamic predictions, our validation and test sets consist of the function to predict for a particular system at a particular temperature. In contrast, the training set contains data for several temperatures for the same system, so to in-
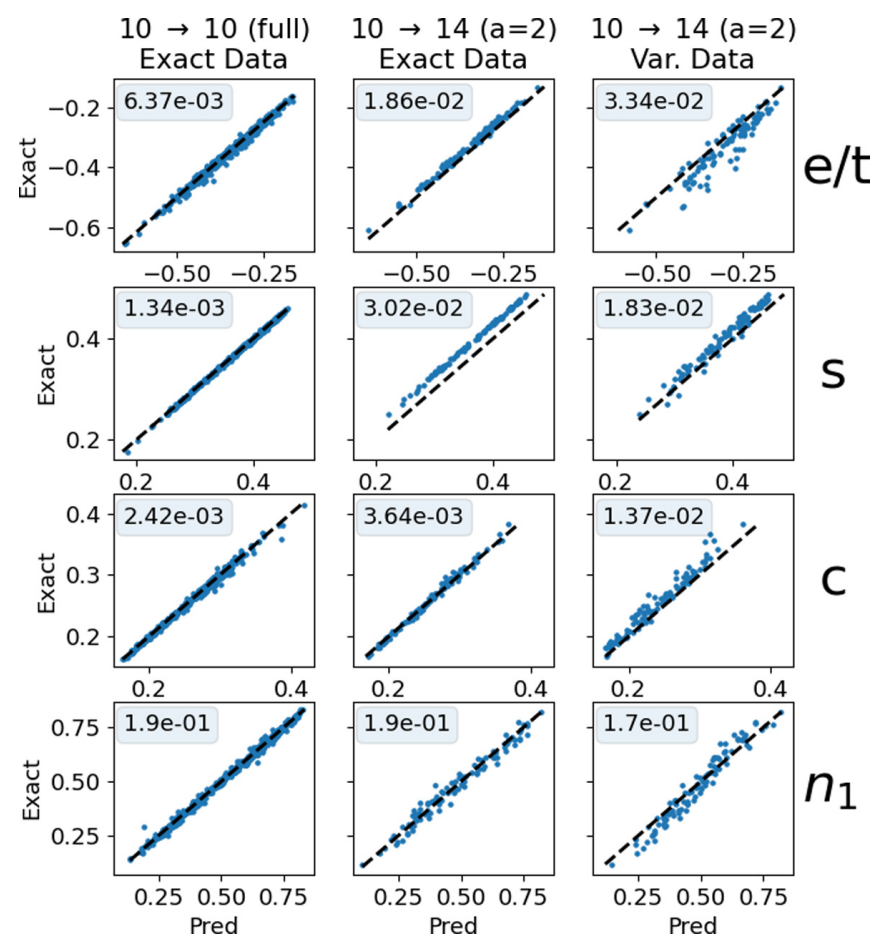

FIG. 5. Demonstration of the one-to-one mapping between ground-state charge density and thermodynamic quantities: $\langle\hat{e}\rangle_{T}$, $\langle\hat{s}\rangle_{T},\langle\hat{c}\rangle_{T}$, and $\left\{\left\langle n_{i}\right\rangle_{T}\right\}$. For $\left\{\left\langle n_{i}\right\rangle_{T}\right\}$ we plot only site $i=1$. Approximated values are plotted against exact results for systems at random temperatures in the range $T \in[1,2]$ obtained with $V=1$ and $W=4$. The left-hand side column is for $L=10$ rings and fully nonlocal models trained on rings of same size. In this case the site occupation is taken from exact diagonalization. The middle column is for semilocal models with $a=2$, where the networks are trained on $L=10$ rings and predict for $L=14$ ones. In this case the groundstate charge density used is the exact one. The right-hand side column depicts results for the same model, but where now the charge density is obtained by variational principle from the ground-state functional. In each panel we report the MAE.

crease the size of the set. The temperatures are randomly chosen between 1 and 2, with the Boltzmann constant set to one (natural units).

Figure 5 shows the results obtained by using this method. In particular, we present a comparison between the MLestimated and the exact quantities for a number of random samples and temperatures, when three different computational strategies are adopted. Data in the left-hand side column are for $L=10$ rings obtained with neural networks trained on rings of the same size. In that case the entire site-occupation vector is used as input, namely, the model is completely nonlocal. The ground-state site occupation used in the ML model is taken from exact diagonalization, namely, it is exact. Thus, this first case aims at understanding whether ML can construct the one-to-one correspondence between ground-state occupation and thermodynamical quantities in the canonical ensemble. In contrast, the middle column shows results for networks trained on $L=10$ rings and predicting the thermodynamics quantities for $L=14$ rings. The locality parameter is chosen to be $a=2$, but the site occupation used to evaluate the various quantity is against the exact one. Thus, this set 
effectively tests the accuracy of the one-to-one mappings. Finally, the left-hand side column is for the same models but now evaluated at the site occupation obtained by minimizing the total energy density with the $f_{V}$ built for the $L=10$ case. Such last case, then explores whether or not a semilocal approximation to the thermodynamics exists and whether this is accessible from the site occupation obtained by variational principle.

From the figure it clearly emerges that ML is indeed able to predict the mapping, and this is significantly more accurate when the ground-state site occupation is exact. A semilocal approximation for such mapping appears to be possible, although the error is certainly larger. In particular, it is clear that there are two main sources of error. The first one is related to the semilocal approximation, so that the thermodynamical quantities computed at semilocal level are less accurate than those for the completely nonlocal model, even when these are evaluated at the exact site occupation (compare the left-hand side column with the middle one). Then there is additional error originating from the use of an approximated site-occupation (compare the middle column with the right-hand side one). Notably, the entropy seems to be the quantity suffering the most from the absence of the correct homogeneous limit in the semilocal models, since the predictions systematically underestimate the exact values.

\section{Construction of the semilocal functional at finite temperature}

Finally, we discuss the extension of ML lattice DFT to finite temperature, namely, we compute the universal functional, $g_{V}$. First, we investigate the locality of the functional by evaluating the MAE as a function of temperature for $a=0$ and $a=1$, when $g_{V}$ is computed at the exact equilibrium siteoccupation, $\left\{n_{i}^{T}\right\}$. Our results for $L=6, V=1$, and $W=4$ are presented in Fig. 6. For each temperature the model has been trained over 6000 samples, validated over 2000, and tested over 2000. Two considerations can be made. On the one hand, a semilocal approximation clearly performs better than a fully local one, which underperforms, in particular at low temperature. On the other hand, the error is significantly reduced as the temperature gets larger and already at $T>6$ little difference is detected between a semilocal and a local approximation. In other words, the functional becomes more local as the temperature increases. This should not be surprising considering that in the limit $T \rightarrow \infty$ the equilibrium density matrix becomes

$$
\hat{\rho}=\frac{1}{\Omega} \mathbb{1}
$$

where $\Omega$ is the dimension of the Hilbert space and $\mathbb{1}$ the identity. This means that for $T \rightarrow \infty$ the equilibrium site occupation is uniform, $n_{i}^{T}=N_{e} / L$, and hence a local and semilocal functionals contain the same information.

Finally, we demonstrate the variational principle for finite temperature DFT with a semilocal functional. Also in this case we train on $L=10$ systems and compute the equilibrium site occupation for rings with 14 sites by gradient-descent minimization (we use exactly the same algorithm as in the $T=0$ case). The starting site occupation is uniform, and the converged one is finally used to compute the Helmholtz

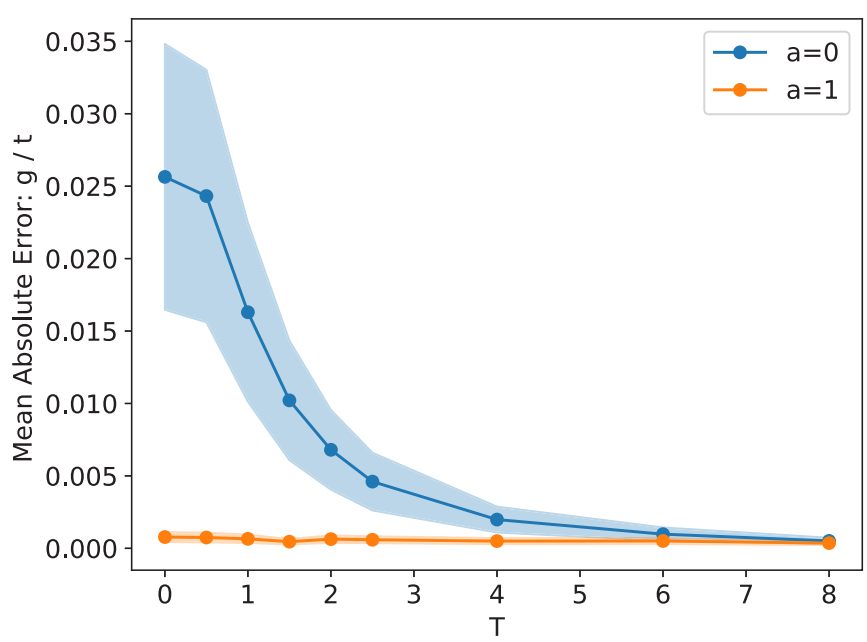

FIG. 6. MAE of the finite-temperature universal functional, $g_{V}$, as a function of $T$ and for different value of the locality parameter, a. Data are presented for $L=6$ rings and parameters $V=1$ and $W=4$. The equilibrium site occupation used to evaluate the functional is that obtained by exact diagonalization. The shaded regions are the standard deviation of the absolute error. For each temperature the training, validation and test sets contain, 6000, 2000, and 2000 samples, respectively.

free-energy density. Figure 7 presents our results for $V=1$, $W=4, a=2$, and temperatures randomly distributed between 1 and 2 .

Clearly, the ML finite-temperature functional appears able to return equilibrium site occupations extremely close to the exact ones (the MAE computed over 100 configurations is 0.029), and good quality Helmholtz free-energy densities. Intriguingly, the Euclidean distance between the converged site occupations and the exact ones is much smaller than in the $T=0$ limit (compare the histograms in Figs. 4 and 7). This reflects two main facts. First, the finite-temperature site occupation is, on average, closer to the homogeneous case than the zero- $T$ one; second, the functional becomes more local at finite temperature. Such tendency towards relatively homogeneous site occupations at high temperatures brings back the issue related to the incorrect homogeneous limit of the semilocal funcitonals, discussed for the $T=0$ case. In fact, the Helmholtz free-energy density appears now systematically underestimated, in particular, for rather uniform densities (see lower panel 4 of Fig. 7). Again the issue can be resolved by either training on larger rings, or by subtracting the noninteracting Helmholtz free-energy density from the functional.

\section{CONCLUSION AND FUTURE WORK}

In this work we have introduced a semilocal approximation to lattice density functional theory for the spinless Hubbard model in one dimension. This has been defined for ground-state DFT, and it has been extended to finite temperature, where the universal functional is constructed for the Helmholtz free-energy density. At the same time, strong of the Hohenberg-Kohn theorems, we have evaluated a number of one-to-one mappings between the ground-state site 

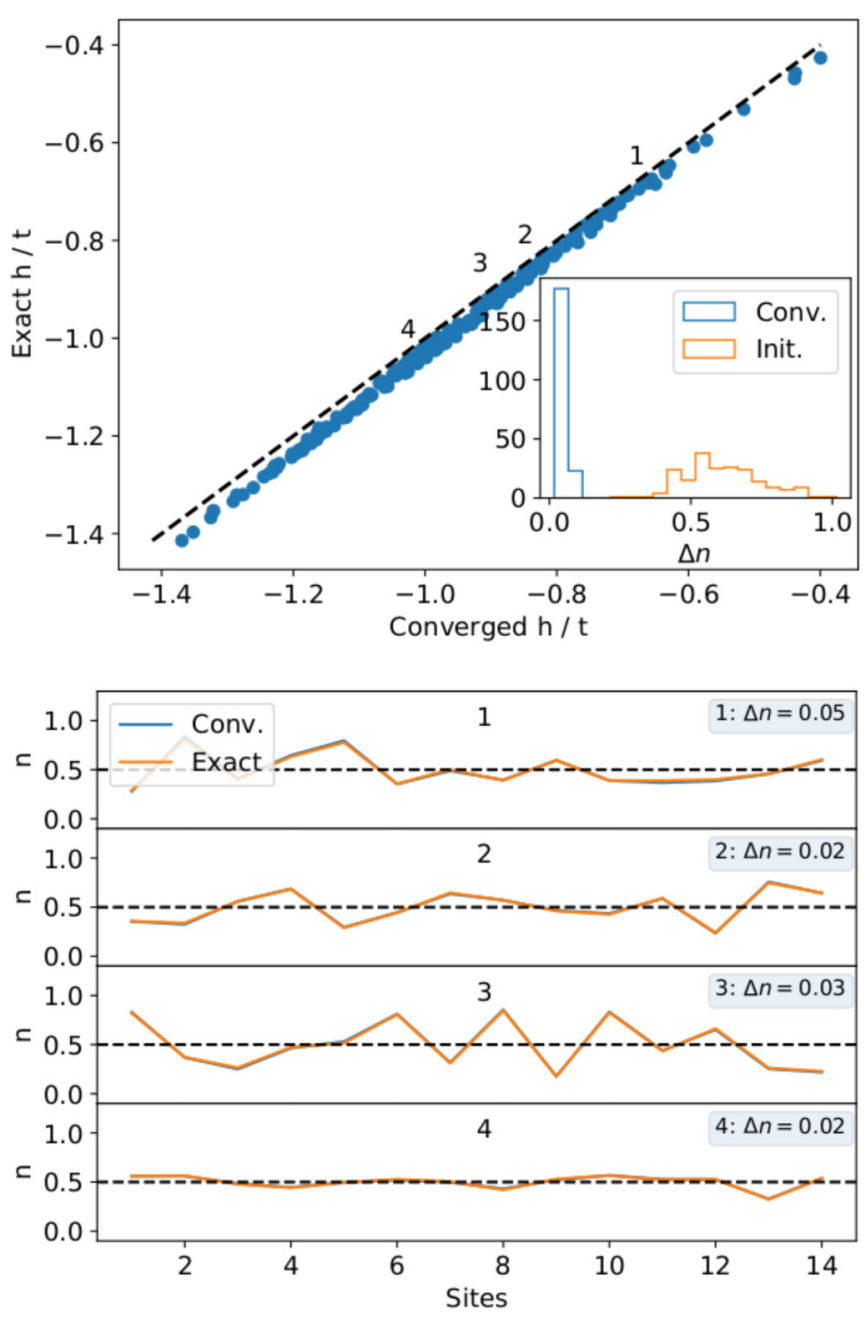

FIG. 7. Demonstration of the variational principle for the finitetemperature ML functional. In the top panel we show the Helmholtz free-energy density computed by minimization with respect to the site occupation. Our network has been trained for $L=10$ and used for a ring containing 14 sites $(V=1)$. The figure contains 100 different disorder realizations with $W=4$. The insert shows histograms of the Euclidean distances $\Delta n$, of the initial and converged equilibrium site occupation from the exact one. The lower panel presents a selection of the converged occupations compared against the exact value. These are taken for different ground-state energy densities as indicated by the numbers in the upper panel. occupation and various thermodynamical quantities at finite temperature. Also in this case a semilocal mapping appears to be possible.

The benefit of constructing such semilocal approximations is that one can train the machine-learning models over systems of moderate size and then use them for much larger ones. In practice, this allows one to explore the thermodynamical limit of various interacting models with disorder. It must be noted that the semilocal approximation defined here does not describe the homogeneous limit, a problem that becomes evident when training on very small systems, or for rather homogeneous exact density. Such problem can be overcome by an appropriate subtraction of the noninteracting energy density (or of the noninteracting Helmholtz free-energy density), or simply by training over systems with adequate size.

The work presented here can extend now to several future directions. First, one can construct similar formalism in two and three dimensions. In this case the dimension of Hilbert space becomes prohibitively large already for small systems and it is likely that it may be no longer possible to generate the training set by exact diagonalization. However, one can alternatively use datasets generated with many-body techniques such as Quantum Montecarlo or other real space approaches (e.g., the Hubbard-Stratonovich approach). A second possibility is to incorporate spin into the representation, namely, consider spin-full models (e.g., the complete Hubbard model). All in all, it appears that machine-learning lattice DFT may become a powerful tool to explore the interplay between interaction and disorder in different dimensions and various lattice models.

\section{ACKNOWLEDGMENTS}

Funding is provided by the Irish Research Council (J.N.) (Grant No. GOIPG/2016/1056) and by the European Research Council project QUEST (R.T.) (Project No. 307891). We acknowledge the DJEI/DES/SFI/HEA Irish Centre for High-End Computing (ICHEC) and Trinity Centre for High Performance Computing (TCHPC) for the provision of computational resources. Special thanks to Dr. Alessandro Lunghi for reading an early draft of the paper.
[1] I. Goodfellow, Y. Bengio, and A. Courville, Deep Learning (MIT Press, Cambridge, MA, 2016), http: //www.deeplearningbook.org.

[2] K. T. Butler, D. W. Davies, H. Cartwright, O. Isayev, and A. Walsh, Nature (London) 559, 547 (2018).

[3] S. Ramakrishna, T.-Y. Zhang, W.-C. Lu, Q. Qian, J. S. C. Low, J. H. R. Yune, D. Z. L. Tan, S. Bressan, S. Sanvito, and S. R. Kalidindi, J. Intell. Manuf. 30, 2307 (2019).

[4] A. Zhavoronkov, Y. A. Ivanenkov, A. Aliper, M. S. Veselov, V. A. Aladinskiy, A. V. Aladinskaya, V. A. Terentiev, D. A. Polykovskiy, M. D. Kuznetsov, A. Asadulaev, Y. Volkov, A. Zholus, R. R. Shayakhmetov, A. Zhebrak, L. I. Minaeva, B. A.
Zagribelnyy, L. H. Lee, R. Soll, D. Madge, L. Xing et al., Nat. Biotechnol. 37, 1038 (2019).

[5] S. Langner, F. Häse, J. D. Perea, T. Stubhan, J. Hauch, L. M. Roch, T. Heumueller, A. Aspuru-Guzik, and C. J. Brabec, Adv. Mater. 32, 1907801 (2020).

[6] S. Steiner, J. Wolf, S. Glatzel, A. Andreou, J. M. Granda, G. Keenan, T. Hinkley, G. Aragon-Camarasa, P. J. Kitson, D. Angelone, and L. Cronin, Science 363, eaav2211 (2019).

[7] V. L. Deringer, M. A. Caro, and G. Csányi, Adv. Mater. 31, 1902765 (2019).

[8] A. Lunghi and S. Sanvito, Sci. Adv. 5, eaaw2210 (2019). 
[9] E. A. Engel, A. Anelli, A. Hofstetter, F. Paruzzo, L. Emsley, and M. Ceriotti, Phys. Chem. Chem. Phys. 21, 23385 (2019).

[10] J. Nelson and S. Sanvito, Phys. Rev. Materials 3, 104405 (2019).

[11] J. Madsen, P. Liu, J. Kling, J. B. Wagner, T. W. Hansen, O. Winther, and J. Schiotz, Adv. Theo. Simul. 1, 1800037 (2018).

[12] G. Carleo, I. Cirac, K. Cranmer, L. Daudet, M. Schuld, N. Tishby, L. Vogt-Maranto, and L. Zdeborová, Rev. Mod. Phys. 91, 045002 (2019).

[13] H. Saito, J. Phys. Soc. Jpn. 86, 093001 (2017).

[14] J. Ma, P. Zhang, Y. Tan, A. W. Ghosh, and G.-W. Chern, Phys. Rev. B 99, 085118 (2019).

[15] J. Rodriguez-Nieva and M. Scheurer, Nat. Phys. 15, 790 (2019).

[16] H. Y. Kwon, N. J. Kim, C. K. Lee, and C. Won, Phys. Rev. B 99, 024423 (2019).

[17] K. Mills and I. Tamblyn, Phys. Rev. E 97, 032119 (2018).

[18] J. Carrasquilla and R. Melko, Nat. Phys. 13, 431 (2017).

[19] K. Ch'ng, N. Vazquez, and E. Khatami, Phys. Rev. E 97, 013306 (2018).

[20] G. Carleo and M. Troyer, Science 355, 602 (2017).

[21] G. Carleo, K. Choo, D. Hofmann, J. E. Smith, T. Westerhout, F. Alet, E. J. Davis, S. Efthymiou, I. Glasser, S.-H. Lin, M. Mauri, G. Mazzola, C. B. Mendl, E. van Nieuwenburg, O. OReilly, H. Thveniaut, G. Torlai, F. Vicentini, and A. Wietek, SoftwareX 10, 100311 (2019).

[22] S. R. White, Phys. Rev. Lett. 69, 2863 (1992).

[23] W. M. C. Foulkes, L. Mitas, R. J. Needs, and G. Rajagopal, Rev. Mod. Phys. 73, 33 (2001).

[24] E. Gull, A. J. Millis, A. I. Lichtenstein, A. N. Rubtsov, M. Troyer, and P. Werner, Rev. Mod. Phys. 83, 349 (2011).

[25] G. Kotliar, S. Y. Savrasov, K. Haule, V. S. Oudovenko, O. Parcollet, and C. A. Marianetti, Rev. Mod. Phys. 78, 865 (2006).

[26] P. Hohenberg and W. Kohn, Phys. Rev. 136, B864 (1964).

[27] O. Gunnarsson and K. Schönhammer, Phys. Rev. Lett. 56, 1968 (1986).
[28] K. Schönhammer, O. Gunnarsson, and R. M. Noack, Phys. Rev. B 52, 2504 (1995).

[29] J. P. Coe, I. D’Amico, and V. V. França, Europhys. Lett. 110, 63001 (2015).

[30] B. Kalita, L. Li, R. J. McCarty, and K. Burke, Acc. Chem. Research 54, 818 (2021).

[31] J. C. Snyder, M. Rupp, K. Hansen, K.-R. Müller, and K. Burke, Phys. Rev. Lett. 108, 253002 (2012).

[32] L. Li, J. C. Snyder, I. M. Pelaschier, J. Huang, U.-N. Niranjan, P. Duncan, M. Rupp, K.-R. Müller, and K. Burke, Int. J. Quantum Chem. 116, 819 (2016).

[33] L. Li, T. E. Baker, S. R. White, and K. Burke, Phys. Rev. B 94, 245129 (2016).

[34] J. Schmidt, C. L. Benavides-Riveros, and M. A. L. Marques, J. Phys. Chem. Lett. 10, 6425 (2019).

[35] K. Mills, M. Spanner, and I. Tamblyn, Phys. Rev. A 96, 042113 (2017).

[36] K. Ryczko, D. A. Strubbe, and I. Tamblyn, Phys. Rev. A 100, 022512 (2019).

[37] F. Brockherde, L. Vogt, L. Li, M. E. Tuckerman, K. Burke, and K.-R. Müller, Nat. Commun. 8, 872 (2017).

[38] K. Yao and J. Parkhill, J. Chem. Theory Comput. 12, 1139 (2016).

[39] M. Bogojeski, L. Vogt-Maranto, M. E. Tuckerman, K.-R. Müller, and K. Burke, Chemrxiv 8079917 (2019).

[40] L. Zepeda-Núñez, Y. Chen, J. Zhang, W. Jia, L. Zhang, and L. Lin, arXiv:1912.00775.

[41] J. Nelson, R. Tiwari, and S. Sanvito, Phys. Rev. B 99, 075132 (2019).

[42] J. R. Moreno, G. Carleo, and A. Georges, Phys. Rev. Lett. 125, 076402 (2020).

[43] N. D. Mermin, Phys. Rev. 137, A1441 (1965).

[44] K. Mills, K. Ryczko, I. Luchak, A. Domurad, C. Beeler, and I. Tamblyn, Chem. Sci. 10, 4129 (2019).

[45] A. Paszke, S. Gross, F. Massa, A. Lerer, J. Bradbury, G. Chanan, T. Killeen, Z. Lin, N. Gimelshein, L. Antiga et al., in Advances in Neural Information Processing Systems (MIT Press, Cambridge, MA, 2019), pp. 8026-8037. 